\title{
Multiple sialolithiasis of submandibular gland: a case report
}

\author{
Nurwahida, ${ }^{1 *}$ Andri Hardianto, ${ }^{1}$ Kiki A.Rizki ${ }^{2}$
}

\section{Abstract}

Objective: Sialolithiasis is a common disease of the salivary glands. Sialolithiasis occurs mainly in the submandibular gland (80-90\%) and to a lesser extent in the parotid gland (5-20\%). Sialoliths may be single or multiple. Multiple sialolith in the submandibular gland are rare. Seventy to $80 \%$ of cases feature solitary stones; only about $5 \%$ of patients have three or more stones. Patients commonly experience pain and/or swelling.

Methods: A 51-year-old male came to Department of Oral and Maxillofacial Surgery with a swelling and pain at the right submandibular. The panoramic radiograph showed a radiopacity mass with well-defined in right submandibular. The ultrasonographic interpretation showed hypoechoic inhomogeneous mass with multiple calcification. The submandibular gland removal was performed by extraoral approach.

Result: Multiple sialolith in the submandibular gland are rare. A surgical approach for removal of sialolith in the submandibular gland could be intraoral or extraoral. In this case report, we described five stones in the submandibular gland, which were removed by extraoral approach.

Conclusion: There are various methods available for the management of salivary stones. In this case report, removal of the submandibular gland with the stone should still be preferred as the gold standard of treatment and could avoid recurrence of this medical condition.
'Department of Oral and Maxillofacial Surgery, Faculty of Dentistry, Padjadjaran University/ Dr. Hasan Sadikin Hospital, Bandung, Indonesia

${ }^{2}$ Department of Head and Neck Oncology, Faculty of Medicine, Padjadjaran University/Dr. Hasan Sadikin Hospital, Bandung, Indonesia

"Correspondence to: Nurwahida, Department of Oral and Maxillofacial Surgery, Faculty of Dentistry, Padjadjaran University/Dr. Hasan Sadikin Hospital, Bandung, Indonesia idh4.nurwahida@gmail.com

Received: 24 April 2017 Revised: 25 April 2017 Accepted: 21 June 2017 Available online: 01 August 2017

Keywords: Sialolithiasis, Multiple, Submandibular gland

Cite this Article: Nurwahida, Hardianto A, Rizki KA. 2017. Multiple sialolithiasis of submandibular gland: a case report. Journal of Dentomaxillofacial Science 2(2): 129-132. D0I: 10.15562/jdmfs.v2i2.530

\section{Introduction}

Sialolithiasis is a most common salivary gland disease. Submandibular gland disease are most prevalent in mid-age with incidence of 12 in 1000 of adult population. This disease affects men twice more than women. ${ }^{1,2}$ The sialolithiasis generally occurs in the submandibular glands with prevalence of more than $80 \%$, about $6 \%$ in the parotid gland and $2 \%$ in the sublingual gland or minor salivary glands. Sialolith can be either single or multiple stones. The prevalence of multiple sialolith in submandibular gland are rare. ${ }^{2}$ Approximately $70-80 \%$ of cases are solitary stone, only about $5 \%$ of cases that have three or more stones in the salivary glands. ${ }^{1}$ Salivary gland stones are generally unilateral and does not cause dry mouth. Clinically, salivary stones are round or oval, rough or smooth and yellowish. ${ }^{2}$

Sialolith stone comprisesof less amount of calcium phosphate compare to carbonate in the form of hydroxyapatite, which is also lesser than magnesium, potassium and ammonia. ${ }^{1-3}$ The composition of submandibular glands stone consists of $82 \%$ inorganic and $18 \%$ organic materials, and the composition of parotid gland stones consist of $49 \%$ inorganic and $51 \%$ organic materials. The organic material is composed of a various carbohydrates and amino acids. But bacterialelementswerenot found in the core of sialolith. ${ }^{2}$
The pathogenesis and etiology of salivary stones is poorly understood. ${ }^{2,4}$ Other than the composition of the saliva, some anatomical factors of submandibular glands and ducts are important in the pathogenesis of stone formation. The submandibular salivary alkaline $\mathrm{pH}$ can also support the formation of salivary stones. ${ }^{4}$

\section{Case Report}

In October 10th, 2014, a 51 years old male patient came to Oral and Maxillofacial Surgery polyclinic with chief complaint of lump and pain on his right lower jaw. The lump had occurred since three years before, first occurred with a size of table tennis ball, the size was often shrinking. The complaint was accompanied with pain during meal time or while chewing. There was history of toothache a month prior to hospital admission. There was no history of weight loss. History of other lump in other part of the body was denied. History of the same complaint in family was also denied. To treat his condition, the patient came to Rajawali hospital. Where he was given analgesic and antibiotic. Due to no improvement, the patient was referred to Hasan Sadikin Hospital.

In general physical examination, there was no abnormalities found. Respiratory rate was 


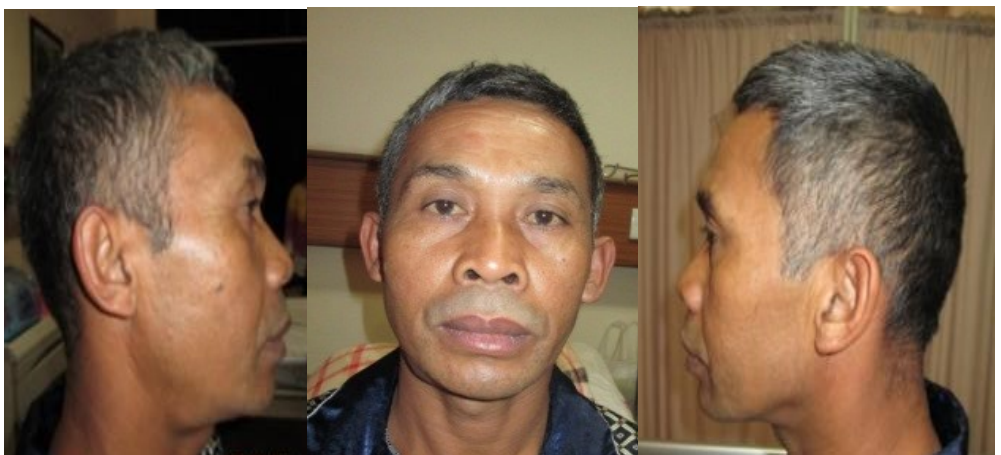

A

B

C

Figure 1 Clinical photograph of the patient, A. Right side view, B. Frontal view, C. Left side view

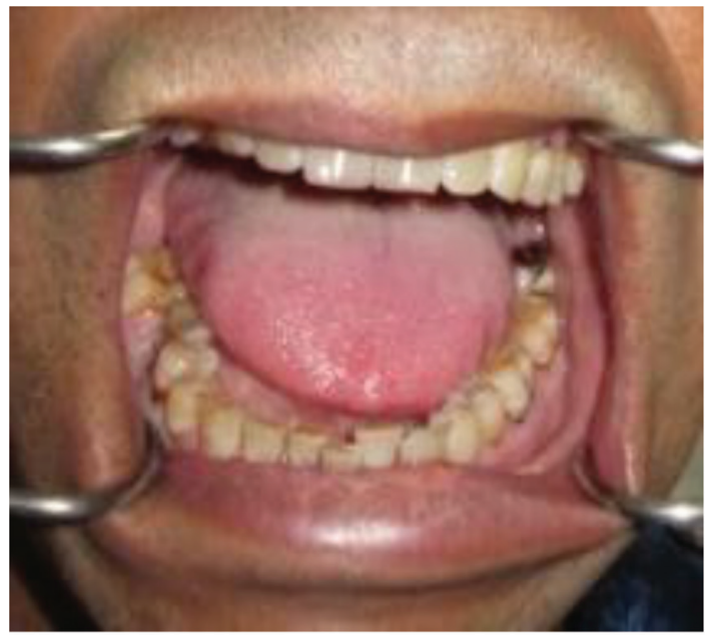

Figure 2 Intra oral photograph

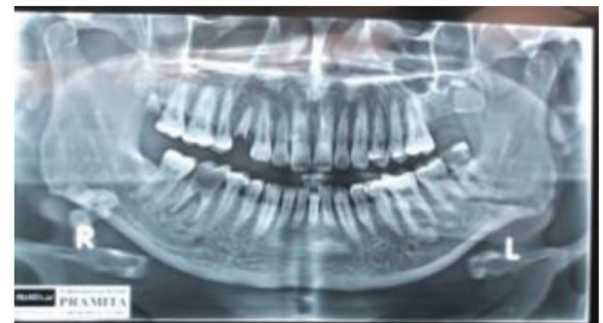

A

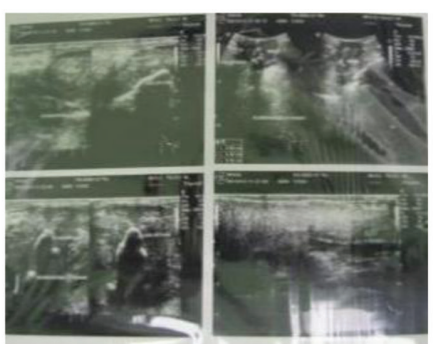

B

Figure 3 A. Panoramic Radiograph, B.Ultrasonography

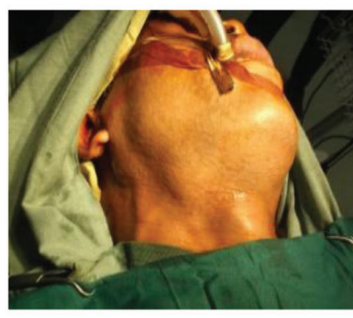

A

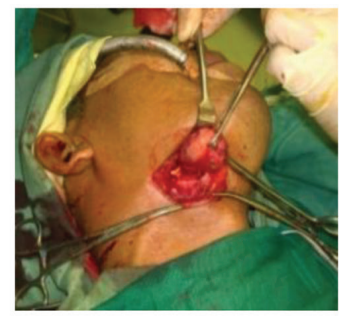

B
18 times/minutes, blood pressure check was $130 / 90 \mathrm{mmHg}$ and pulse was 82 times/minutes.

Alocal extra oral examination presenteda symmetrical face figure $1 \mathrm{~B}, 1 \mathrm{C}$, with lump in right lower jaw, the color of the skin was similar to surrounding tissue figure $1 \mathrm{~A}$. Through palpation on extra oral, the lump hada hard consistency, non-febrile temperature, uneven surface, fixated, and no tenderness, with $4 \times 3 \times 3 \mathrm{~cm}$ in size figure $1 \mathrm{~A}$.

Intraoral examination found gangrene radices of teeth 46,38 and 14 . No visible abnormalities were found in buccal mucosa, gingiva, tongue and floor of the mouth figure 2. There was noenlargement of lymph nodes found on submandibular glands and neck examination.

In laboratory tests, hematology and clinical chemistry was within normal limits.In radiology, the panoramic view showeda radiopaque mass with firm boundaries in the right submandibular region figure 3A. On ultrasonography (USG) of the neck, there was hypoechoic and inhomogeneous mass accompanied by multiple calcifications with $4.0 \times 3.46 \times 3.33 \mathrm{~cm}$ size in the medial region of the parotid gland figure $3 \mathrm{~B}$.

On October 23rd. 2014, surgical treatment was conducted to remove the right submandibular gland. Incision was done on the right submandibular region and continued with removal of right submandibular gland and retrieval of 5 sialolith stones. The flap closure was done by suturing the muscle and the skin layer-by-layer and then followed with drain installation and cleaning the surgery area. A press bandage was appliedon the post-sutured site figure 4 .

Patient was discharged on the second day after surgery figure $5 \mathrm{~A}$ then he was scheduled to control at the polyclinic of oral and maxillofacial surgery on November 7th 2014 figure 5B bringing the result of anatomic pathology tissue examination. Microscopically tissue sample was found to be coated. The subepitheliumshowedstromal fibro-collagen connective tissue, powdered massive inflammatory cells: lymphocytes, eosinophil, and plasma cells. Salivary gland presence among the cells with tubular shape coated with thorax

Figure 4 A. Incision site preparation, B. Removal of right submandibular gland and retrieval of sialolith stones, C. Flap closure with suturing and drain application, D. The excised submandibular gland and 5 sialolith stones 


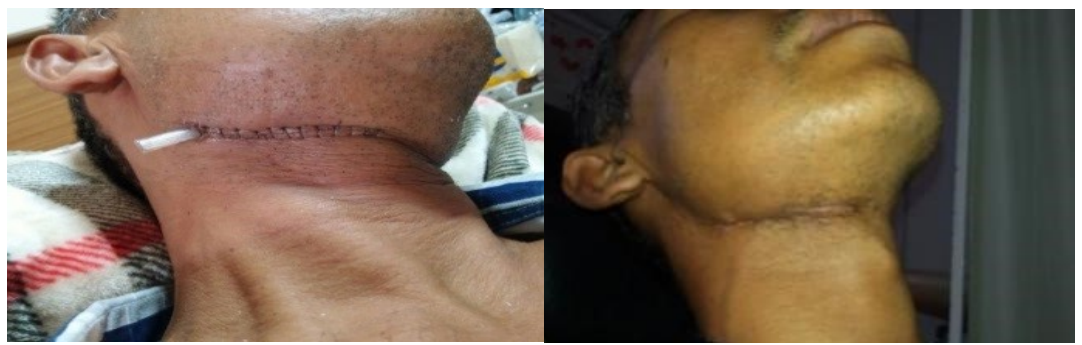

A

B

Figure 5 A. Post-operative of the second day, B. Control after 2 weeks

epithelium, the cells' nucleus were within normal limits. The ducts of salivary gland weredilated. A subcapsularview presence hyperplastic lymphoid follicles with dilating blood vessels accompanied with bleeding area. Also present aremature fat cells, with nucleus on the edge of the cells was within normal limit. There was no sign of malignancy. So histologically it was to be concluded as chronic nonspecific sialadenitis sialolithiasis at submandibular region.

\section{Discussion}

Sialolithiasis is a common salivary gland disease..$^{1,2,5}$ The sialolithiasis generally occurs in the submandibular glands with prevalence of more than $80 \%$, and $6 \%$ in the parotid gland and $2 \%$ in the sublingual gland or minor salivary glands. ${ }^{2}$

Clinical manifestations of submandibular stones will appear more clearly at mealtime, duringthe time of maximum saliva production, causing a sudden swelling and is usually very painful. ${ }^{2,5} \mathrm{~A}$ clinician should record the symptoms likepain, lumps, decreased salivary flow, and malaise. ${ }^{6-8}$ Swelling and pain that recurs in the gland can be associated to food or when seeing foods. The swelling reduced gradually, but will be repeated every time when the salivary flow is stimulated. . $^{1,2,5,6}$ This process may continue until the complete obstruction, infection, or a combination of both. Obstruction, with or without infection, may lead to atrophy of the involved glandular secretion cells. ${ }^{8}$ Glands infection manifests with swelling in the floor of the mouth, erythema and associated cervical lymphadenopathy. ${ }^{5}$ The period and duration of the swelling are very helpful in diagnosis. Clinical examination and palpation of the gland can help determine the lump in the gland, fixation, ulceration, or local nerve involvement and the intraoral examination can assess the decrease or increase of the salivary flow. ${ }^{1,6}$

Radiographs often show radiopaque stones. To examine the submandibular gland, the panoramic or occlusal photograph can be useful to see the gland ducts. Dental panoramic photograph show both areas of submandibular glands and parotid glands, but the definitive diagnosis usually requires more specific examination. ${ }^{6}$

Ultrasonography is a simple method that can indicate a sialolith with high accuracy. But sialolith that are smaller than $3 \mathrm{~mm}$ cannot be detected with ultrasound. ${ }^{1}$

There are several techniques used for sialolith stone removal, which depends on the duration of symptoms, number of recurrent episodes, the size of the stone, and perhaps most importantly the location of the stone. ${ }^{3,5,9}$ Small sialolith, located near the peripheral ducts can be removed by manipulation (called flushing gland). Largesialolith are removed by surgery. Sometimes, the stone that is not affected can be extracted through the duct intubation using a soft plastic catheter and the application of suction to the tube. Multiple stones or stones in the salivary gland requires the removal of the gland itself. ${ }^{3}$

Almost half of the submandibular gland stones is located in the distal third of the submandibular duct and a simple surgery through an incision can be done at the floor of the mouth, which issimple and usually not associated with complications. If the salivary stoneis located anterior enough, the stone can be massaged and manipulated through the duct hole with probe lacrimal guidance and a dilator to open the duct. Once open, the stone can be identified and removed. The glands are then massaged to remove other debris in the posterior part of the duct.The opening of the duct is required to remove the stone. It involves a transoral approach where the incision is made directly into the stone location. Through this approach, a more posterior stone/stones can be removed by cutting directly on the longitudinal axis of the duct. The wound closure was not done for the purpose of drainage.If the gland has been damaged by recurrent infection and fibrosis or if stone been has formed in the salivary gland, then it requires the removal of the salivary gland itself., ${ }^{2,6}$ Complications that may occur are scar tissue, facial nerve paralysis, or paresthesia of the lingual. ${ }^{6}$

\section{Conclusion}

Sialolithiasis is a common salivary gland disease. However, the prevalence of multiple sialolith in the submandibular gland is rare. Clinical manifestations of submandibular stones can beclearly noticed at the meal time, when the saliva production increases, which causesa sudden swelling and is usually very painful. The swelling will reduce gradually, but it will be repeated everytime when the salivary flow is stimulated. There are several 
methods of management that are used in the removalthe salivary gland stones. Handling of the submandibular gland stones depends on the duration of symptoms, number of recurrent episodes, stone size and location of the stone. In this case, the removal of the submandibular gland is still the best option in the management of multiple sialolith and can avoid possible recurrences.

\section{Conflict of Interest}

The authors report no conflict of interest.

\section{References}

1. Sunder VS, Chakravarthy C, Mikkilinine R, et al. Multiple bilateral submandibular gland sialolithiasis. Niger J Clin Pract 2014;17: 115-118.

2. Siddiqui SJ. Sialolithiasis: an unusually large submandibular salivary stone. Br Dent J 2002;193: 89-91.
3. Mouli C, Kumar SM, Kailasam S, et al. Sialolith: a case report with review of literature. Indian J Multidiscip Dent 2011;1: 377-379.

4. Grasesa F, Santiagob C, Simoneta BM, et al. Sialolithiasis: mechanism of calculi formation and etiologic factors. Clin Chim Acta 2003;334: 131-136.

5. James RH, Edward EI, Myron RT. Contemporary oral and maxillofacial surgery. 6th ed. Elsevier Mosby; 2014. p. $405-408$.

6. Moore UJ. Principles of oral and maxillofacial surgery. 6th ed. Blackwell Publishing Ltd; 2011. p. 306-308.

7. Daniel ML. Clinician's handbook of oral and maxillofacial surgery. 1st ed. Chicago: Quintessence; 2010. p. 363-366.

8. Dalkiz M, Dogan N, Beydemir B. Sialolithiasis (salivary stone). Turk J Med Sci 2001;31: 177-179.

9. Tucker AS, Miletich I. Salivary glands: development, adaptations and disease. J Anat. 2010;217: 755-756.

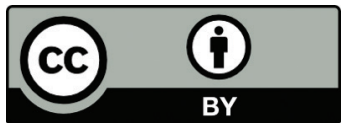

This work is licensed under a Creative Commons Attribution 